\title{
El apego. Más allá de un concepto inspirador.
}

Attachment: Further an inspiring concept.

\author{
Antonio Galán Rodríguez ${ }^{\text {a }}$
}

${ }^{a}$ Doctor en Psicología - Psicólogo Clínico. Servicio de Atención y Protección a la Infancia y Adolescencia de la Junta de Extremadura

Correspondencia: Antonio Galán Rodríguez, (antonio.galan.rodriguez@juntaextremadura.es)

Recibido: 19/02/2010; aceptado: 18/04/2010

RESUMEN: La Teoría del Apego ha proporcionado conceptos fundamentales para explicar las relaciones humanas; no obstante, el uso que se hace de esta teoría a menudo es muy simple y no respeta sus ideas centrales. Para ilustrarlo, se analizan tres aspectos teóricos a tener en cuenta: la clasificación de los tipos de apego, la transmisión intergeneracional de los patrones de apego, y los trastornos del apego. También se revisan tres importantes cuestiones a clarificar en relación al apego: su correcta definición, su uso en contextos aplicados (a nivel de evaluación y tratamiento), y los límites del concepto. Finalmente, el apego es situado en un contexto más amplio donde la intersubjetividad ocupa una posición central para comprender la conducta humana.

PALABRAS CLAVE: Apego, Apego desorganizado, Brecha de la transmisión, Trastornos del apego, Función reflexiva.
ABSTRACT: Attachment Theory has provided important concepts to explain human relationships; nevertheless, the use of this theory is often very simple and doesn't respect its key concepts. Three theoretical aspects to take into consideration are analyzed: the attachment classification, the transmission of attachment patterns along the generations, and the attachment disorders. Also, three important opened matters about attachment are reviewed: the right definition of the concept, its use in practical settings (assessment and treatment), and the limits of the concept. Finally, attachment is set in a wider context where intersubjectivity occupies a main place to understand human behavior.

KEY WORDS: Attachment, Disorganized attachment, Transmission gap, Attachment disorder, Reflexive function.

\section{El apego: ¿marco conceptual o concepto inspirador?}

En el ámbito de la intervención clínica y psicosocial es frecuente recurrir a la Teoría del Apego para explicar la relación entre un niño y sus cuidadores. John Bowlby propuso una concepción relacional del ser humano (el niño viene al mundo preparado para establecer un estrecho lazo de unión con una figura de cuidado) sostenida por un marco conceptual de gran interés $(1,2,3)$. Las aportaciones de Mary Ainsworth permitieron la expansión (conceptual y académica) de la Teoría del Apego, consolidando así lo que parecía una fructífera y prometedora línea de trabajo (4).

No obstante, un análisis detenido de la presencia de la Teoría del Apego en contextos aplicados nos sitúa frecuentemente ante usos vagos, inapropiados o restrin- 
ORIGINALES Y REVISIONES

gidos, que no soportarían un contraste riguroso con los fundamentos de este marco conceptual. Además, es frecuente limitar su aplicación a la propuesta teórica básica, y desconsiderar así las numerosas contribuciones posteriores que nos muestran la fertilidad de este campo de conocimiento.

De esta manera, parece que asistimos al contraste entre: a) una línea de investigación consistente y fructífera; y b) una simplificación por la que el concepto de "apego" aparece más como un concepto inspirador de una forma de entender al ser humano, que como un marco de referencia con todas sus implicaciones.

El objetivo de este trabajo es señalar algunos ámbitos en los que con más facilidad puede caerse en una lectura confusa o simplificadora de la Teoría del Apego. No se trata por tanto de una introducción a este marco teórico, para lo que remitimos a las obras de Marrone (5) o de Yarnov (6), como de una actualización centrada en los retos, dificultades y propuestas que puedan ayudarnos a extraer todo su potencial.

\section{Algunos aspectos teóricos sobre los que incidir.}

Existen ámbitos de la Teoría del Apego donde se aprecia con claridad cómo una visión simplificada implica la pérdida de un importante potencial explicativo. La limitación de espacio nos obliga a realizar una selección de tres.

2.1. Los tipos de apego.

La forma habitual de clasificar los tipos de apego se asienta en la propuesta de Ainsworth, al distinguir entre apegos seguro, evitativo y resistente-ambivalente. La descripción de estos se basa en la respuesta de los niños a un procedimiento de laboratorio conocido como "Situación Extraña", en la que el infante es sometido a una serie de separaciones y reencuentros con su figura de apego. Hay niños que se dirigen con rapidez a la madre cuando vuelve y se sienten a gusto con ella ("apego seguro"); otros no muestran malestar al quedarse solos, y evitan o ignoran a la madre en el reencuentro ("apego evitativo"). Finalmente, algunos niños no se alejan de la madre, protestan enérgicamente por la separación, y no llegan a calmarse en el reencuentro ("apego ambivalente-resistente").

El error más habitual en el que puede caer el profesional es esperar una reproducción literal de esas respuestas, y esto ocurre porque no se tienen en cuenta el contexto en que se elaboró esta tipología. El estudio partió de las observaciones de la interacción niño-madre que Ainsworth recogió en Uganda entre 1954 y 1955. Tras la estancia coyuntural en ese país africano y su definitiva instalación en los EEUU, trató de replicar los hallazgos a través del "Estudio Baltimore", donde recogió observaciones de interacciones en el hogar para 26 diadas madre-hijo. En esta investigación, las observaciones en el laboratorio mediante el Procedimiento de Situación Extraña 
ORIGINALES Y REVISIONES

aparecían como un complemento a los registros en el hogar. Por ello, los auténticos resultados del estudio surgían de contrastar las observaciones en ambos contextos. Y así podemos entender, por ejemplo, que para caracterizar al niño con apego evitativo no debemos esperar una conducta continua de indiferencia hacia la madre (la que aparece en la Situación Extraña), porque en el hogar estos niños se mostrarán ansiosos y enfadados, manifestando malestar incluso ante separaciones muy breves; una posible explicación es que ante el estrés de la separación, el niño opta por desactivar el sistema de apego (7). Por tanto, la respuesta en el contexto de laboratorio era un potente indicador de un patrón de apego, pero no aportaba una muestra de la conducta esperable en el niño en cualquier entorno.

Datos como éste derivan en una reivindicación de la observación naturalista en el hogar, y alertan de los peligros de abordar una evaluación del apego exclusivamente en contextos artificiales (de laboratorio de investigación, o de despacho clínico). De hecho, la misma Ainsworth protestaba por el uso generalizado de la Situación Extraña a expensas de ese otro tipo de observación (8).

Otra consecuencia indeseable de tomar con excesiva literalidad las descripciones del Procedimiento de Situación Extraña es que no se tiene en cuenta el factor evolutivo. En efecto, dicha estrategia de evaluación se desarrolló para niños que tenían alrededor de un año de edad. Y dado que la manifestación de las estrategias de apego cambia conforme el niño crece, la descripción que aporta la Situación Extraña, en cuando conductas visibles, no deben ser las esperadas en otros momentos del crecimiento. Por ejemplo Crittenden (9) plantea que a los 12 meses se distingue entre apego seguroambivalente-evitativo, mientras que a los 21 o 36 meses la distinción se establecerá entre seguro-coercitivo-defensivo. Es por esta plasticidad de la conducta que algunos teóricos del apego han intentado encontrar una continuidad en las manifestaciones del apego, entendiendo éste como constructo organizacional que integra el desarrollo en sus distintos ámbitos (10); de esta manera, el apego ejercería un efecto continuo durante el crecimiento, si bien sus manifestaciones externas cambiarán a lo largo del desarrollo.

Finalmente, un tercer error vinculado a la tipología de apego es que con frecuencia tiende a ser considerada casi como una clasificación de personalidad, atribuyendo a sus manifestaciones unos rasgos de permanencia y generalización que no responden a la realidad. Debemos tener en cuenta que ya en el mismo momento de la evaluación, el niño puede mostrar un tipo de apego diferenciado según el progenitor de que se trate (por ejemplo, seguro con la madre y evitativo con el padre). De la misma manera, el niño puede presentar cambios en el tipo de apego con lapsos de tiempo relativamente cortos. Todo ello apunta a un carácter dinámico y contextual del apego, que lleva a considerar éste en términos de estrategias de adaptación más que en el de características del individuo.

En la clasificación tripartita original de Ainsworth no aparecía un tipo de apego que más tarde acabaría generando una ingente bibliografía y cuyas implicaciones se 
ORIGINALES Y REVISIONES

muestran especialmente relevantes a nivel teórico y aplicado. A principios de los 80, algunos investigadores, y especialmente los que trabajaban con población maltratada, informaban de las dificultades para clasificar a algunos niños. Finalmente, y con la ayuda de Judith Solomon, la investigadora Mary Main identificó este nuevo patrón de apego, al que denominó "desorganizado/desorientado" (11). En situaciones donde se activa el sistema de apego, el comportamiento característico de estos niños sería: a) presentación secuencial o simultánea de conductas contradictorias; b) movimientos y expresiones indirectas, mal dirigidas, incompletas, e interrumpidas; c) movimientos estereotipados, asimétricos o mal temporalizados, y posturas anómalas; d) quedarse paralizado o moverse lentamente; e) índices indirectos de aprehensión respecto al progenitor; f) índices directos de desorganización y desorientación. Se trata por tanto de manifestaciones especialmente dramáticas, que sugieren la existencia de dificultades serias en los procesos de estructuración interna del niño y del vínculo entre éste y sus cuidadores.

La primera teorización acerca del apego desorganizado vinculaba éste a un miedo sin solución, a la existencia de un dilema insoluble al que se estaría enfrentando el niño; en efecto, su conducta no sería incoherente o bizarra, sino indicadores de que vive una experiencia de angustia que no puede resolver porque el cuidador es la fuente de miedo al mismo tiempo que constituye el único puerto potencial de seguridad. Esto explicaría su frecuente aparición en familias con un padre maltratador (casi 80\% de los niños de estas familias). Lyons-Ruth amplió esta visión original, sugiriendo que el apego desorganizado también puede ser el producto de un padre extremadamente insensible o alterado que falla continuamente en tranquilizar y confortar la activación en el niño de las necesidades de apego (12); por tanto, el niño quedaría expuesto a la disregulación emocional sin contar con la ayuda de su cuidador. Main resumía estas situaciones hablando de cuidadores aterrorizados, aterrorizantes, y disociativos (7).

Esta propuesta de apego desorganizado merece atención, tanto por las posibles interpretaciones mal dirigidas, como por el potencial que está mostrando a la hora de aportar luz sobre algunos ámbitos de intervención. Empezando por las primeras, debemos volver a mencionar el peligro de considerar un tipo de apego como un rasgo de personalidad. De hecho, es posible que con el apego desorganizado ni tan siquiera pueda hablarse de una forma de apego, en cuanto que podría ser más propiamente el resultado del colapso en las estrategias de apego; es decir, que cuando el niño no es capaz de regular sus emociones vinculadas al apego usando las estrategias habituales, aparecería la desorganización (11). Esto explicaría su aparición breve y transitoria, que obliga a contar con un buen entrenamiento para identificarlo (13) y nos permite entender por qué transcurrió tanto tiempo hasta que pudo ser descrito por los investigadores. No obstante, también es cierto que a veces la desorganización es tan predominante, que no puede detectarse una estrategia secundaria organizada, de manera que el niño mantiene de forma persistente una conducta de apego seriamente alterada. 
ORIGINALES Y REVISIONES

Continuando con posibles asunciones erróneas, no debemos esperar que el comportamiento del niño tal como fue descrito anteriormente sea la única manifestación de un apego desorganizado. De hecho, los estudios de seguimiento han probado la existencia de un cambio importante ya a los 3-4 años de vida, cuando la desorganización se convierte en control. Es decir, en un determinado momento se produce una inversión de rol, por la que el infante trata de implantar las normas de la relación, y lo hará a través de una estrategia coercitiva (imposición, violencia, chantaje sobre los progenitores) o de cuidado (extrema solicitud, rol de cuidador sobre los padres). Hesse y Main señalan que en estos casos la desorganización permanece en el nivel de las representaciones mentales (lo que se reflejará en los dibujos, en sus narrativas...) mientras que a nivel conductual aparecen nuevas conductas, las de coerción o excesiva acomodación (11). De esta manera, el adolescente o adulto controlador, agresivo, incapaz de auto-calmarse cuando se enfrenta con tormentas emocionales o pérdidas, propenso a la disociación e incapaz de apartarse de relaciones que provocan dolor, puede estar mostrándonos la evolución de un apego desorganizado (14).

Respecto a las implicaciones acerca de esta forma de apego, nos gustaría destacar dos ámbitos de investigación especialmente interesantes. En primer lugar, su relación con la psicopatología. Parece suficientemente acreditado el vínculo entre apego desorganizado y manifestaciones psicopatológicas posteriores $(10,13,14)$. A partir de lo que señalábamos anteriormente, se entiende la relación con muchos trastornos del comportamiento en niños, adolescentes y jóvenes. Finalmente, uno de los ámbitos más fértiles a nivel de investigación se encuentra en el estudio de la relación entre apego desorganizado infantil y los trastornos disociativos y borderlines en el adulto $(15,16,12)$.

Por otro lado, el apego desorganizado ha recibido una especial atención en los ámbitos familiares donde fue identificado, y en los que su presencia es más frecuente: los contextos de pobreza, desestructuración, marginalidad... Así, mientras que en familias normales de clase media aparece en aproximadamente el $15 \%$ de los niños, en estos otros contextos sociales y en grupos clínicos, el porcentaje puede incrementarse hasta el doble o el triple (13). Esto ha generado una importante bibliografía en torno a la especificidad de esos contextos desfavorecidos. La aportación más sobresaliente es el Minnesota Longitudinal Study of Parents and Children, un ambicioso estudio longitudinal liderado por L. Alan Sroufe, que comenzó en los 70 y que aún continúa $(10,17)$.

2.2. Reactualización adulta del apego y transmisión intergeneracional.

El interés por el lazo existente entre las experiencias de vinculación de los padres y el apego que muestran los hijos tiene un gran interés teórico y aplicado, de ahí que se haya convertido en una de las líneas de trabajo más abordadas. Gran parte de las investigaciones aparecen ligadas a un instrumento de evaluación, la Adult Attachment Interview (AAI). Se trata de una entrevista semiestructurada que gira alrededor de 15 cuestiones y que trata de evaluar el estado mental del sujeto en relación a su his- 
ORIGINALES Y REVISIONES

toria de vinculaciones. Algunas personas valoran con claridad a las figuras y las experiencias de apego, con una aparente objetividad, y en un discurso equilibrado; estos rasgos configuran la categoría "Seguro/autónomo". Otros sujetos ofrecen un discurso pobre, donde tienden a no reconocer o discutir los eventos negativos, especialmente su significación emocional, y ofreciendo un discurso ("memoria semántica") que no encuentra suficiente apoyo en los hechos ("memoria episódica"); estaríamos ante la categoría "Despreocupado". Otras personas parecen demasiado preocupadas con las relaciones tempranas y/o actuales como para describirlas y evaluarlas con claridad, ofreciendo discursos muy largos pero vagos y confusos; se trataría de la categoría "Preocupado". Finalmente, algunos individuos muestran desorganización o desorientación en el discurso o el razonamiento cuando intentar discutir eventos traumáticos (declaraciones incompatibles o que violan las relaciones espaciotemporales o causales, cambios abruptos en el hilo del discurso...); se trataría de la categoría "No resuelto-desorganizado". Es importante tener en cuenta que la clave de la AAI no es lo que el sujeto cuenta sino cómo lo hace (calidad, cantidad, relevancia y forma de la comunicación).

A pesar de lo exigente en cuanto a formación y uso, la AAI ha sido profusamente utilizada (18). Ha permitido entender la forma en que el adulto organiza su historia de apego, y cómo influye en sus vinculaciones actuales; de hecho, a pesar de su carácter de instrumento de investigación, también es utilizada en la clínica (19). No obstante, aquí nos centraremos en uno de los datos que aportó este instrumento en alguno de los primeros estudios, cuando permitió apreciar que no eran los eventos de las vidas de los padres lo que permitía predecir el tipo de vínculo de sus hijos; era el grado en que aquellos habían integrado y dado sentido a sus experiencias lo que determinaba la seguridad del apego en los hijos. En efecto, se encontraba un paralelismo entre la clasificación de apego infantil (seguro, evitativo, ambivalente y desorganizado) y el tipo de apego en sus padres según la AAI (seguro-autónomo, despreocupado, preocupado y desorganizado-no resuelto, respectivamente) Este hallazgo sería reforzado por el de Fonagy, Steele y Steele (20) en el conocido como London Parent-Child Project, al evaluar a 100 embarazadas con el AAI y posteriormente a la diada madre-hijo con el Procedimiento de Situación Extraña cuando el niño había cumplido un año. Incluso con esta evaluación prenatal, la representación mental de la madre de su historia de apego permitía predecir el tipo de apego que en el futuro tendría su hijo.

Pero la pregunta que surge a continuación es "¿Cómo se establece esta continuidad entre las experiencias de vinculación de los progenitores y el apego de los hijos?". Es lo que en la literatura recibe el nombre de "la brecha de la transmisión" ("the transmission gap"), un campo en el que aún no hay resultados concluyentes, pero cuyo interés a nivel aplicado es enorme; en efecto, si descubrimos los elementos que permiten la transmisión de formas problemáticas de apego, podremos orientar 
ORIGINALES Y REVISIONES

con mayor eficiencia las intervenciones; de hecho, el campo de trabajo que ha recibido más atención es el que uniría la categoría "No resuelto" de la AAI con el apego desorganizado del niño. En algún momento se piensa en términos más conductuales, como las prácticas de crianza (véase por ejemplo el metaanálisis de Madigan, Bakermans-Kranenburg, Van Ijzendoorn, Moran, Pederson y Benoit (21)). En otros, se abordan cuestiones más sutiles que tienen que ver con la intersubjetividad que se establecería en la diada madre-hijo; entre ellos destaca los conceptos de "mentalización" y "función reflexiva". Estos han adquirido una relevancia central en la Teoría el Apego, y algunos estiman que han pasado a jugar un papel central en la teorización actual sobre el desarrollo del niño, quizá comparable al que jugó el "complejo de Edipo" en los inicios del Psicoanálisis. Surgen de la propuesta de Peter Fonagy a partir del ya mencionado London Parent-Child Study. Al analizar los datos aportados por éste, pensando en los mecanismos que explicarían la transmisión intergeneracional del apego, los investigadores llegaron a captar la importancia que tenía la capacidad de la madre para pensar en su hijo en términos psicológicos; se trata de la posibilidad de sostener en su propia mente una representación del niño como alguien que tiene sentimientos, deseos e intenciones propias; y será esta capacidad materna lo que permita predecir el tipo de apego que acabará presentando su hijo (22).

2.3. Los trastornos del apego.

La categorización de los trastornos del apego constituye en la actualidad unos de los ámbitos de mayor interés aplicado entre los profesionales (véase por ejemplo el monográfico de Attachment \& Human Development de 2003); no obstante, encontramos aquí uno de los campos relativos al apego donde aparecen más incertidumbres, y también en el que más se han dado casos de mala comprensión y praxis.

En efecto, aunque encontramos bastante acuerdo entre los clínicos e investigadores en que existen formas alteradas o patológicas de apego, aún no contamos con un planteamiento ampliamente aceptado acerca de su clasificación y definición. La propuesta más extendida es la que aparece en el DSM como "trastorno reactivo de la vinculación de la infancia o la niñez", con planteamientos similares en la CIE y la Clasificación Diagnóstica 0-3. Incluye unos síntomas relativos a la conducta de apego, y los liga a un causa concreta (maltrato o crianza en ambientes que limitan la oportunidad de formar apegos selectivos). No obstante, existe cierto descontento con esta propuesta $(23,24)$. Por ejemplo, se señala el carácter restringido de su planteamiento, al basarse en la conducta social en contextos muy determinados (niños maltratados e institucionalizados); pero además, aparece como uno de los trastornos menos investigados y donde más faltan trabajos sistemáticos de investigación. También se ha planteado alguna objeción a la distinción entre las formas inhibida y desinhibida. Por ejemplo, Zeanah y Smyke (25) plantean que podrían tratarse de problemas diferentes en lugar de dos presentaciones de un mismo problema. Es más, señalan que el patrón desinhibido podría no ser un trastorno del apego; alegan que es 
ORIGINALES Y REVISIONES

posible encontrar niños adoptados que sí llegan a establecer un lazo de apego con el cuidador pero que siguen manteniendo una conducta de relación desinhibida con los demás adultos.

Ante estas limitaciones se han presentado propuestas alternativas. Por ejemplo, Boris y Zeanah (23) plantean un espectro que recogería las distintas formas de presentación del trastorno de apego. En un extremo estaría el apego seguro, pasaría a formas ordinarias de apego inseguro (evitativo y resistente), continuaría con el apego desorganizado, luego las Distorsiones de Base Segura, y finalmente los Trastornos del Desapego, que se aproximan a los trastornos de apego. No obstante, existen argumentos en contra de este tipo de continuum (26). Por nuestra parte, nos planteamos si en realidad habría que considerar dos dimensiones: a) la formación o no de un lazo de apego, lo que depende de la presencia y estabilidad de un cuidador, y que podría implicar en los casos extremos un trastorno reactivo de la vinculación (tal como ocurre en los casos de severas institucionalizaciones); y b) las características del apego, que tienen que ver con la calidad del cuidado, y que daría lugar a los distintos tipos de apego problemático; respecto a estos últimos, apenas se ha avanzado en el intento de describirlos y clasificarlos. En este punto es necesario anotar que los apegos evitativo y ambivalente no deberían ser considerados un problema psicopatológico, a pesar de que frecuentemente son descritos como tales. Esto podría ser diferente en el caso del apego desorganizado; en efecto, es tal su relación con una futura deriva psicopatológica, que se ha llegado a plantear el conceptuarlo como una manifestación temprana de psicopatología (13).

Estas dudas acerca de la definición y clasificación de los trastornos del apego están relacionadas en gran medida con la falta de conocimientos acerca de estos. Gran parte de los estudios se han realizado con niños criados en instituciones, y resulta cuestionable que los hallazgos en esta población puedan ser generalizados directamente a cualquier contexto de crianza. A pesar del valor de dichos estudios, tanto a nivel aplicado (han permitido una atención especializada a algunos colectivos) como teórico (nos han mostrado una imagen descarnada de las distorsiones graves en los procesos de apego), las generalizaciones pueden ser científica y éticamente cuestionables. La objeción ética procede del peligro de estigmatizar a ciertos niños cuando se establece una identidad entre maltrato (o institucionalización) y trastorno del apego. Es evidente que un cuidado severamente inapropiado puede implicar una disfunción en los procesos de apego, pero no existe un determinismo en esta asociación. Por otra parte, a nivel metodológico debemos tener en cuenta que, si la definición del trastorno reactivo de la vinculación se ha basado en la observación de una población muy determinada, es de esperar un sobrediagnóstico de ésta.

Otro importante problema deriva de una excesiva generalización del concepto de "trastorno de apego", al convertir éste en el eje de una intervención que debería estar dirigida en otra dirección. En efecto, el carácter sugerente de la Teoría del Ape- 
ORIGINALES Y REVISIONES

go ha llevado a numerosos profesionales a aplicar en exceso este modelo explicativo; y así, donde otros planteamientos teóricos o aplicados podrían ser más adecuados, se está interpretando la conducta del niño desde la óptica del apego (27). De ahí la recomendación de considerar los trastornos del apego como la segunda opción diagnóstica, en cuanto que muchas dificultades que experimentan los niños podrían ser mejor entendidas con las categorías nosológicas más consolidadas. Este problema ha recibido una especial atención dentro de la polémica que han generado algunas prácticas profesionales que se amparan bajo el rótulo de "terapias de apego", y cuyo estatus científico y ético ha sido duramente cuestionado por colectivos profesionales como la American Professional Society on the Abuse of Children (24); en relación al tema que nos ocupa, se critica el hecho de calificar como "trastorno del apego" cualquier caso donde aparezcan dificultades en la relación padres-hijo. Todo ello abunda en la necesidad de extremar el rigor científico y ético al incorporar la Teoría del Apego a la práctica profesional.

Finalmente, se debería mencionar al menos dos aportaciones más de interés en esta línea, y que son valiosas porque implican líneas de trabajo muy productivas. En primer lugar, el valor de las guías clínicas para la intervención ante los problemas relativos al apego, como la de la American Academy of Child and Adolescent Psychiatry (AACAP) (28), pero de las que sigue habiendo cierta escasez. En segundo lugar, debemos tener en cuenta que se pueden reinterpretar diversos cuadros psicopatológicos desde la Teoría del Apego. Ya el propio Bowlby lo hizo con algunos de ellos, como las fobias (2) y los procesos de duelo (3). Estas relecturas son fundamentales para entender estos procesos psicopatológicos (véase por ejemplo, a Tizón (29) para el duelo).

\section{Algunos cuestionamientos y propuestas.}

La fructífera expansión de la Teoría del Apego que acabamos de revisar no oculta la existencia de lagunas, problemas y asuntos pendientes, algunos de las cuales ya hemos ido apuntando. En este apartado trataremos de reflejar varias más, además de incluir propuestas que permitan un avance de este campo de conocimiento. Nuevamente, por limitaciones de espacio, nos limitaremos a tres cuestiones que consideramos representativas.

3.1. La imprecisión en las definiciones.

El término "apego" se ha acabado convirtiendo en un amplio paraguas que intenta abarcar todo tipo de relación emocional; de esta manera, se establece una identidad entre "apego" y "vínculo afectivo", en lugar de considerar a aquél como uno más de los posibles lazos emocionales que pueden establecerse entre dos seres humanos 
(30). Éste es uno de los grandes errores existentes en nuestro entorno, donde se habla de "apego" sin la adecuada precisión terminológica y conceptual. Frente a ello, Ainsworth $(8,30)$ limitaba las predicciones basadas en el apego infantil al ámbito de las relaciones íntimas. En la misma línea, Bowlby planteaba que el apego debería recoger sólo el lazo emocional con alguien percibido como mayor o más sabio (31). Tras estas propuestas aparece una búsqueda del elemento que caracterizaría a la relación de apego; probablemente el concepto central sea el de búsqueda de seguridad recurriendo a una figura poderosa, y cuyo origen filogenético residiría en la protección frente a depredadores, las agresiones de congéneres o la posibilidad de no seguir al grupo en movimiento (7). Por ello, la evaluación del apego podría ser realmente predictiva cuando se aborda en contextos de peligro, no en las interacciones cotidianas, puesto que es en aquellos donde el apego aparece activado. Esta clarificación conceptual debería ir acompañada de indicadores comportamentales o emocionales que permitan identificar con seguridad cuándo existe una relación basada en el apego, tal como hacen Parrish (32) o Liotti (15).

3.2. El apego en contextos aplicados.

El campo de la evaluación y la intervención desde la Teoría del Apego ha experimentado un gran desarrollo, a pesar de lo cual persisten importantes dificultades, de entre las cuales se pueden señalar algunas.

Respecto a la evaluación, no se abordará aquí una revisión de los instrumentos (véase para ello por ejemplo Yarnov (33)); además, por falta de espacio no entraremos a considerar la tradición proveniente de la Psicología Social y de la Personalidad, que extiende las ideas básicas de la Teoría del Apego al estudio de las relaciones amorosas y del apego, incidiendo especialmente en el apego adulto. Centrándonos en la evaluación del apego infantil (en niños y adultos), encontramos aquí un gran desarrollo, pero acompañado de una falta de proyección en el ámbito aplicado. En efecto, algunos instrumentos de evaluación permanecen muy anclados en los contextos de investigación; otros se han mostrado útiles en contextos aplicados, pero su excesiva complejidad (por la formación que requieren del profesional o por el tiempo que demandan en su aplicación) limita seriamente su uso. Todo esto se traduce en un desfase entre la frecuencia con la que se usa el concepto de apego en la intervención profesional, y la evaluación estandarizada que se hace de él. Una afirmación muy explícita (y quizá algo exagerada) en este sentido la hace Nilsen (27) refiriéndose específicamente a la atención a los jóvenes; este autor plantea que muchos profesionales obvian las propuestas de la Teoría del Apego planteándose para qué intentar evaluar lo que no se puede definir de forma fiable y para lo no que no se tienen herramientas efectivas de intervención. No obstante, hay propuestas para sistematizar la evaluación clínica (34) o adaptar algunos de los procedimientos de investigación al ámbito aplicado, como ha ocurrido con la Situación Extraña en la propuesta recogida por la American Academy of Child and Adolescent Psychiatry (28). No obstante, aún debe avanzarse mucho más en esta línea. 
ORIGINALES Y REVISIONES

Respecto a la aplicación de la Teoría del Apego al ámbito terapéutico, advertimos de cierta falta de sistematización. Las propuestas de Bowlby implicaban entender la tarea psicoterapéutica de una forma diferente (el terapeuta como "base de seguridad", importancia de las experiencias reales de cuidado...); de hecho, el propio Bowlby introdujo cambios en su trabajo clínico, aunque sorprendentemente no llegó a formular una propuesta terapéutica diferenciada (35). A duras penas podríamos hablar de una terapia del apego propiamente dicha, en el sentido de una propuesta derivada directamente de la Teoría del Apego, y con carácter diferenciado respecto a otros enfoques. Lo que encontramos es un amplio rango de posibilidades en cuanto a la medida en que sus presupuestos han sido incorporados al quehacer terapéutico. Así, en algún caso el concepto de apego se limita a aparecer como un elemento de sensibilización a las relaciones de cuidado; en otros, la Teoría del Apego se presenta como un complemento a propuestas de otro tipo (conductuales, estructurales...), apareciendo como una contribución extraordinaria ante casos o situaciones especialmente difíciles $(36,37)$. No obstante, en otros casos el apego se convierte en un vértice de gran importancia en el diseño del formato terapéutico. Por ejemplo, el concepto de mentalización ha dado lugar a formatos específicos de tratamiento (38) o al énfasis en ciertas herramientas en la acción terapéutica (39). Finalmente, debe subrayarse cómo en los últimos años asistimos al desarrollo de diversos modelos teóricos que enfatizan los aspectos intersubjetivos del desarrollo humano, y cuyos presupuestos muestran una gran afinidad con la Teoría del Apego, de modo que existe una complementariedad o solapamiento entre estas diversas conceptuaciones de la relación padres-hijos. Nos referimos a propuestas muy extendidas como las de Stern (40) o Trevarthen (41), con conceptos como los de "entonamiento", "constelación maternal" o "intersubjetividad". Esta confluencia se ha traducido en que algunos formatos terapéuticos que se consideran fundamentados en la Teoría del Apego, en realidad estén incluyendo éste dentro de una propuesta más amplia, tal como ocurre en la Child-Parent Psychotherapy (42) o en la Dyadic Developmental Psychotherapy $(43,44)$.

3.3. Los límites del concepto.

Los padres asumen muchas funciones en el cuidado de sus hijos, y no todas se enmarcan en el apego. Guiar y poner límites, socializar la expresión emocional, o apoyar las relaciones con los pares, se sitúan en un marco diferente al de proporcionar seguridad en un contexto de cuidado. Una muestra de esta complejidad la encontramos en el estudio Minnesota; éste se centró originalmente en el concepto de apego, pero acabó derivando en un teoría del desarrollo, tratando de explicar las complejas y dinámicas relaciones entre todos los elementos que configuran la evolución del ser humano (10). Tal como indica Vaugh (17), una relación de base segura co-construida en la diada niño-padre no puede explicar todo el comportamiento humano, y esto es así porque los senderos de la infancia a la adaptación adulta casi nunca son planos y simples; se complican y deben ser comprendidos dentro del contexto de ambientes 
anidados en la diada, la familia, la comunidad y la cultura, con las que el individuo se maneja en mayor grado conforme crece.

Ahora bien, si no todo es apego, ¿cómo se relaciona éste con el resto de componentes del vínculo? Estimamos que clarificar esta cuestión es uno de los grandes retos a los que se enfrenta actualmente la Teoría del Apego. Se trata de un desafío ya abordado en los distintos modelos multidimensionales de la motivación humana que han incluido el apego, como los de Lichtenberg (45), Bleichmar (46) o Liotti (15); no obstante, estas propuestas en absoluto agotan la complejidad de esas interrelaciones entre el apego y el resto de los procesos que sustentan las relaciones humanas.

Pero estos interrogantes acaban derivando en un cuestionamiento de la propia identidad del concepto, ya que el apego es incluido en propuestas más globales acerca del funcionamiento psíquico. Se trata de planteamientos que reconocen el valor inmediato del apego (asegurar una relación de cuidado), pero también una utilidad más amplia (crear un molde para interpretar las relaciones de cuidado, o servir como modelo de regulación emocional) que llega a su máxima extensión al considerar el papel que jugaría en los procesos nucleares de la estructuración psíquica (crear el marco en el que surge la intersubjetividad). Por ello, un fallo en los procesos de apego tendrá consecuencias a muy diversos y comprometidos ámbitos de funcionamiento personal; podrá influir en la forma en que el individuo busca seguridad en situaciones estresantes (el foco de interés en las primeras formulaciones del apego), pero también en el acceso a la capacidad para establecer una conexión emocional consigo mismo y con los demás. El interés por este último aspecto es el que lleva a autores como Lyons-Ruth (16) a proponer un cambio del énfasis desde los mecanismos de la proximidad física y la protección hasta los del intercambio intersubjetivo. En esta línea se sitúan también aportaciones como la de Fonagy $(22,47)$, al postular que el apego es el marco interaccional en el que se desarrollan los mecanismos cerebrales que permiten los procesos básicos de autoregulación (reacción al estrés, capacidad para mantener focalizada la atención, capacidad para interpretar los estados mentales propios y de los demás); es decir, las relaciones de apego que Bolwby describió, y que nos insertaban en el mundo de las especies animales, constituirían también el marco general en el que se desarrollarían algunos de los procesos básicos más propiamente humanos.

\section{Más allá del apego.}

La evolución histórica de la Teoría del Apego nos sitúa ante progresivos niveles de profundidad a la hora de conceptualizar las relaciones humanas, y el profesional puede optar (en función de sus intereses o conocimientos) por visiones más o menos 
ORIGINALES Y REVISIONES

complejas de ese vínculo. La propuesta básica de Bowlby incidía en los aspectos más observables y evidentes de la conducta, y más adelante fue dando cabida a componentes de carácter cognitivo, anticipando lo que acabaría siendo denominado el "giro representacional". Los avances posteriores en la definición del concepto, en su elaboración teórica, y en el desarrollo de instrumentos de investigación, han permitido enriquecer la visión del apego, iluminando nuevos aspectos de los vínculos humanos. Finalmente, parece que en la actualidad asistimos a un nuevo salto conceptual al enmarcar el apego dentro de un ámbito más amplio del desarrollo humano. En concreto, es situado en el contexto más global de los procesos que configuran la intersubjetividad, entendiendo ésta como una capacidad humana básica que nos permite leer los estados de los otros y compartir aspectos de nuestras vidas mentales. Esto implica una reformulación de los conceptos básicos de la Teoría del Apego, y de cómo integremos este "giro intersubjetivo", dependerá en gran medida las posibilidades de avance de este campo de conocimiento.

BIBLIOGRAFÍA:

(1) Bowlby J. El apego y la pérdida 1. El apego. Barcelona: Paidós, 1998

(2) Bowlby J. El apego y la pérdida 2. La separación. Barcelona: Paidós, 1998.

(3) Bowlby J. El apego y la pérdida 3. La pérdida. Barcelona: Paidós, 2004

(4) BRETHERTON I. The origins of attachment theory: John Bowlby and Mary Ainsworth. Dev Psychol 1992; 28: 759-775.

(5) Marrone M. La Teoría del Apego. Un enfoque actual. Madrid: Psimática, 2001.

(6) YARNov S, EDITOR. La Teoría del Apego en la clínica, I. Evaluación y clínica. Madrid: Psimática, 2008

(7) MaIN M. The organized categories of infant, child, and adult attachment: flexible vs. inflexible attention under attachment-related stress. J Am Psychoanal Assoc 2000; 48: 1055-1096.

(8) Ainsworth M, MarVin RS. On the shaping of attachment theory and research: an interview with Mary D.S. Ainsworth. Monogr Soc Res Child Dev 1995; 60: 3-20.

(9) CRitTEnden PM. Nuevas implicaciones clínicas de la teoría del apego. Valencia: Promolibro, 2002.

(10) Sroufe LA. Attachment and development: a prospective, longitudinal study from birth to adulthood. Attach Hum Dev 2005; 7: 349-367.

(11) Hesse E, MaIN M. Disorganized infant, child, and adult attachment: collapse in behavioral and attentional strategies. J Am Psychoanal Assoc 2000: 48: 1097-1127.

(12) Lyons-Ruth K. Contributions of the mother-infant relationship to dissociative, borderline, and conduct symptoms in young adulthood. Infant Ment Health J 2008: 29: 203-218.

(13) Van IJzendoorn MH, Schuengel C, BaKermans-Kranendurg MJ. Disorganized attachment in early childhood: Meta-analysis of precursors, concomitants, and sequelae. Dev Psychopathol 1999; 11: 225-249. 
(14) HoLmES J. Disorganized attachment and borderline personality disorder: a clinical perspective. Attach Hum Dev 2004; 6: 181-190.

(15) Lıтті G. La relación terapéutica con el paciente borderline: un análisis en términos de desorganización del apego. En Yarnov S, editor. La Teoría del Apego en la clínica, I. Evaluación y clínica. Madrid: Psimática, 2008; p. 163-186.

(16) Lyons-Ruth K. The interface between attachment and intersubjectivity: perspective from the longitudinal study of disorganized attachment. Psychoanalytic Inquiry 2006; 26: 595-616.

(17) VAUGH BE. Discovering pattern in developing lives: reflections on the Minnesota study of risk and adaptation from birth to adulthood. Attach Hum Dev 2005; 7: 369-380.

(18) Backermans-Kranerburg MJ, Van IJzendoorn MH. The first 10,000 Adult Attachment Interviews: distribution of adult attachment representations in clinical and non-clinical groups. Attach Hum Dev 2009; 11: 223-263.

(19) BuchHeIm A, Kächele H. Adult Attachment Interview and Psychoanalytic Perspective: a single case study. Psychoanlytic Inquiry 2003; 23: 81-101.

(20) Fonagy P, Steele H, Steele M. Maternal representations of attachment during pregnancy predict the organization of infant-mother attachment at one year of age. Child Dev 1991; 62: 891-905.

(21) Madigan S, Bakermans-Kranenburg MJ, Van IJzendoorn MH, Moran G, Pederson DR, BENOIT D. Unresolved states of mind, anomalous parental behavior, and disorganized attachment: a review and meta-analysis of a transmission gap. Attach Hum Dev 2006; 8: 89-111.

(22) Fonagy P. Teoría del apego y Psicoanálisis. Barcelona: Espaxs, 2004.

(23) Boris NW, ZEANAH CH. Disturbances and disorders of attachment in infancy: an overview. Infant Ment Health J 1999; 20: 1-9.

(24) Chaffin M, Hanson R, Saunders BE, Nichols T, Barnett D, Zeanah C, et al. Report of the APSAC Task Force on Attachment Therapy, Reactive Attachment Disorder, and Attachment Problems. Child Maltreat 2006; 11: 76-89.

(25) Zeanah CH, Smyke AT. Attachment disorders in family and social context. Infant Ment Health J 2008; 29: 219-233.

(26) O'CONNOR TG, ZEANAH C. Current perspectives on attachment disorders: rejoinder and synthesis. Attach Hum Dev 2003; 5: 321-326.

(27) NILSEN WJ. Perceptions of attachment in academia and the child welfare system: the gap between research and reality. Attach Hum Dev 2003; 5: 303-306.

(28) American ACAdemy of ChILd and Adolescent Psychiatry (AACAP). Practice parameter for the assessment and treatment of children and adolescents with Reactive Attachment Disorder of infancy and early childhood. J Am Acad Child Adolesc Psychiatry 2005; 44: 1206-1219.

(29) Tizón JL. Pérdida, pena, duelo: vivencias, organización y asistencia. Barcelona: Paidós, 2004.

(30) Ainsworth M. Attachments beyond infancy. Am Psychol 1989; 44: 709-716.

(31) Stevenson-Hinde J. Attachment theory and John Bowlby: some reflections. Attach Hum Dev 2007; 9: 337-342.

(32) EAGLE M. Clinical implications of Attachment Theory. Psychoanlytic Inquiry 2003; 23: 27-53.

(33) Yarnov S. La evaluación desde la Teoría del Apego: el lugar de los autoinformes y otros instrumentos en la evaluación del apego en niños, adolescentes y adultos. En Yarnov S, editor. La Teoría del Apego en la clínica, I. Evaluación y clínica. Madrid: Psimática, 2008; p. 95-161. 
ORIGINALES Y REVISIONES

(34) Boris NW, Fueyo M, Zeanah CH. The clinical assesment of attachment in children under five. J Am Acad Child Adolesc Psychiatry 1997; 36: 291-293.

(35) TROWELL J. Reflections on 'an attachment-based treatment of maltreated children and young people'. Attach Hum Dev 2004; 6: 279-283.

(36) Diamond GS, LidDLE HA. Transforming negative parent-adolescent interactions: from impasse to dialogue. Fam Process 1999; 38: 5-26.

(37) GiRón S. Toxicomanías: integración de la Teoría del Apego y la de Sistemas. Revista de la Sociedad Europea de Profesionales con Intervención en Drogodependencias 2002; 6(2): 9-32.

(38) SLADE A. Reflective Parenting Programs: Theory and Development. Psychoanalytic Inquiry 2006; 26: 640-657.

(39) Tyson P. Research in Child Psychoanalysis: twenty-five year follow-up of a severely disturbed child. J Am Psychoanal Assoc 2009; 57: 919-945.

(40) STERN DN. La constelación maternal. Barcelona: Paidós, 1997

(41) Trevarthen C, AitKen KJ. Infant intersubjectivity: research, theory, and clinical applications. J Child Psychol Psychiatry 2001; 42: 3-48.

(42) LIEBERMAN AF. Ghosts and angels: intergenerational patterns in the transmission and treatment of the traumatic sequelae of domestic violence. Infant Ment Health J 2007; 28: 422-439.

(43) Becker-Weidman A, Hughes D. Dyadic Developmental Psychotherapy: an evidence-based treatment for children with complex trauma and disorders of attachment. Child and Family Social Work 2008; 13: 329-337.

(44) Hughes D. An attachment-based treatment of maltreated children and young people. Attach Hum Dev 2004; 6: 263-278.

(45) Lichtenberg JD, Schonbar RA. Motivation in Psychology and Psychoanalysis. En Barron JW, Eagle MN, Wolitzky DL, editors. Interface of Psychoanalysis and Psychology. Washington D.C.: APA Books, 1992; p. 11-36.

(46) Bleichmar H. Avances en Psicoterapia Psicoanalítica. Barcelona: Paidós, 1997.

(47) Fonagy P, TARgEt M. Early intervention and the development of self-regulation. Psychoanalytic Inquiry 2002; 22: 307-335. 